\title{
Altmetrics and Other Novel Measures for Scientific Impact
}

\author{
Martin Fenner
}

Good science, original work, always went beyond the body of received opinion, always represented a dissent from orthodoxy. However, then, could the orthodox fairly assess it?

-Richard Rhodes mod. from Michael Polanyi in "Making of the Atomic Bomb"

\begin{abstract}
Impact assessment is one of the major drivers in scholarly communication, in particular since the number of available faculty positions and grants has far exceeded the number of applications. Peer review still plays a critical role in evaluating science, but citation-based bibliometric indicators are becoming increasingly important. This chapter looks at a novel set of indicators that can complement both citation analysis and peer review. Altmetrics use indicators gathered in the real-time Social Web to provide immediate feedback about scholarly works. We describe the most important altmetrics and provide a critical assessment of their value and limitations.
\end{abstract}

\section{Introduction}

Impact assessment of researchers and their research is central to scholarly communication. In the last 25 years, we have seen a shift from individual qualitative assessment by peers to systematic quantitative assessment using citation analysis of journal articles. Arguably the impact of research can not be quantified, and citation analysis falls short of a comprehensive analysis, but the journal as a filter for relevant scholarly content and the Journal Impact Factor as a tool to quantify the relevance of journals are at the core of how research is communicated and evaluated today.

The central role of the journal (to distribute, filter, and help evaluate scholarly content) has dramatically changed with the shift from print to electronic publishing, and is no longer appropriate for the assessment of impact. We can look at

\footnotetext{
M. Fenner ( $₫)$

Public Library of Science, San Francisco, CA, USA

e-mail: mfenner@plos.org
} 
citations of individual articles, and at other measures of impact using usage stats and the Social Web. Moreover, impact assessment does not have to be confined to journal articles; research outputs such as data publication can also be assessed.

Altmetrics is a young discipline that looks at new metrics based on the Social Web for analyzing scholarship. Altmetrics are complementary to the citationbased filters which we have relied upon for the past 50 years and try to overcome some of their shortcomings: citations are slow to accumulate, and often miss new forms of scholarly content such as datasets, software, and research blogs (Priem et al. 2012a).

Altmetrics are challenging this established system, and are therefore seen by many as either an opportunity or a threat to the current system of scholarly communication. This potential makes altmetrics both fascinating and challenging, as many discussions about altmetrics are often intermixed with other ideas about how to change scholarly communication.

\section{Terminology}

Scientometrics is the science of measuring and analysing science.

Bibliometrics is a major subdiscipline of scientometrics which measures the impact of scientific publications. Citation analysis is the most popular application of bibliometrics.

Usage-based metrics use usage data (pageviews, document downloads, etc.) to assess scholarly impact. The concept was popularized by the COUNTER (Counting Online Usage of NeTworked Electronic Resources) and MESUR (MEtrics from Scholarly Usage of Resources) projects.

Altmetrics is the creation and study of new metrics based on the Social Web for analyzing and informing scholarship (Altmetrics Manifesto ${ }^{1}$ ). Altmetrics is a subdiscipline of scientometrics. Altmetrics typically looks at individual research outputs, including journal articles or datasets.

Article-level metrics are a comprehensive and multidimensional suite of transparent and established metrics at the article level. ${ }^{2}$ They collect and provide metrics for individual articles, rather than aggregating them per journal. Articlelevel metrics include citations, usage data, and altmetrics. Article-level metrics are typically associated with the publisher Public Library of Science (PLOS), who

\footnotetext{
1 Altmetrics: http://altmetrics.org/about

2 PLOS Article-Level Metrics: http://article-level-metrics.plos.org/alm-info/
} 
introduced them for all of their articles in 2009. Altmetrics and article-level metrics are sometimes used interchangeably, but there are important differences:

- Article-level metrics also include citations and usage data

- Altmetrics can also be applied to other research outputs, such as research data

Metrics for other research works - presentations, datasets, software, etc.-typically include usage statistics and altmetrics, but also citations.

Author-level metrics aggregate the metrics of all research by a specific author. Metrics can also be aggregated by institution, discipline, etc.

Post-publication peer review is the process whereby scientific studies are absorbed into the body of knowledge (Smith 2011). This definition is much broader and does not just include activities that are traditionally described as peer review. In contrast to metrics, the focus is on the discussion of a paper in comments, blog posts, and citations. A broader term with similar meaning is postpublication activity.

\section{History}

In 2008 Dario Taraborelli published a paper on soft peer review, advocating social bookmarking tools for post-publication peer review (Taraborelli 2008). Neylon and Wu described the PLOS Article-Level Metrics service launched in 2009 in an article published the same year (Neylon and Wu 2009). Priem and Hemminger published an article in July 2010 that describes scientometrics 2.0 and called for new metrics based on Web 2.0 tools (Priem and Hemminger 2010). Groth and Gurney studied chemistry science blogging about scholarly papers and presented their findings at the Web Science Conference 2010 (Groth and Gurney 2010). The Altmetrics manifesto was published in October 2010 by Jason Priem, Dario Taraborelli, Paul Groth and Cameron Neylon (Priem et al. 2010).

ReaderMeter is a web service that tracks the number of Mendeley readers of all papers of a particular author. ReaderMeter was launched in late 2010 and is the first working altmetrics service. The first altmetrics workshop was was altmetrics11, held at the ACM Web Science Conference 2011 Workshop $^{3}$ in June 2011. Hackathons are an important part of altmetrics history: a working prototype for Total Impact (now ImpactStory) was put together at the Beyond Impact conference in May 2011, and the idea of the ScienceCard project started at the Science Online London conference in September 2011. Three of the 11 finalists of the Mendeley/ PLOS Binary Battle programming contest in September 2011 were altmetrics applications. In 2012, we saw the launch of several altmetrics services, more publishers implementing altmetrics for their journal articles, and an increasing number of presentations and workshops dedicated to altmetrics.

3 ACM Web Science 2011: http://www.websci11.org/ 


\section{Scholarly Research}

Two workshops dedicated to altmetrics research and associated with the ACM Web Science conference were held: June 2011 in Koblenz, Germany and June 2012 in Evanston, IL.

PLOS ONE launched the Altmetrics collection in October 2012, with initially 7 research articles published since June 2009. ${ }^{4}$

Much early altmetrics research has examined reference managers, particularly Mendeley and CiteULike. Li et al. (2011) found $92 \%$ of Nature and Science articles in their sample had been bookmarked by one or more Mendeley users, and $60 \%$ by one or more CiteULike users. Bar-Ilan (2012) showed $97 \%$ coverage of recent JASIST articles in Mendeley. Priem et al. (2012) reported that the coverage of articles published in the PLOS journals was $80 \%$ in Mendeley and $31 \%$ in CiteULike. Sampling 1,397 F1000 Genomics and Genetics papers, Li and Thelwall (2012) found that 1,389 of those had Mendeley bookmarks.

Studies have consistently found moderate correlation between reference manager bookmarks and Web of Science (WoS) citations. Li et al. (2011) showed $\mathrm{r}=0.55$ of Mendeley and $\mathrm{r}=0.34$ of CiteULike readers with WoS citations respectively. Weller and Peters (2012) report similar correlation values for a different article set between Mendeley, CiteULike, BibSonomy, and Scopus. Bar-Ilan (2012) found a correlation of $r=0.46$ between Mendeley readership counts and WoS citations for articles in JASIST. User-citation correlations for sampled Nature and Science publications were 0.56 ( Li et al. 2011); Priem et al. (2012b) report a correlation of 0.5 between WoS citations and Mendeley users articles published by the Open-Access publisher PLOS.

Twitter has also attracted significant interest from altmetrics researchers. Priem and Costello (2010) and Priem and Costello (2011) report that scholars use Twitter as a professional medium for discussing articles, while Eysenbach (2011) found that highly-tweeted articles were 11 times more likely become highly-cited later. Analyzing the use of Twitter during scientific conferences, Weller and Puschmann (2011) and Letierce et al. (2010) report that there was discipline-specific tweeting behavior regarding topic and number of tweets, as well as references to different document types including journal articles, blogs, and slides. Other sources have examined additional data sources besides reference managers and Twitter, investigating examined citation from Wikipedia articles (Nielsen 2007) and blogs (Groth and Gurney 2010; Shema et al. 2012) as sources of alternative impact data.

${ }^{4}$ PLOS Collections: http://www.ploscollections.org/altmetrics 


\section{Use Cases}

Altmetrics can complement traditional bibliometrics in a number of scenarios:

- Metrics as a discovery tool

- Data-driven stories about the post-publication reception of research

- Business intelligence for a journal, university or funder

- Evaluation of the impact of research and researchers

\section{Metrics as a Discovery Tool}

Information overflow has become a major problem, and it has become clear that relying on the journal as a filter is no longer an appropriate strategy. Altmetrics have the potential to help in the discovery process, especially if combined with more traditional keyword-based search strategies, and with the social network information of the person seeking information. The advantage over citation based metrics is that we don't have to wait years before we can see meaningful numbers. The free Altmetric PLOS Impact Explorer ${ }^{5}$ is an example for a discovery tool based on altmetrics and highlights recently published PLOS papers with a lot of social media activity. Altmetric.com also provides a commercial service for content from other publishers.

\section{Data-Driven Stories About The Post-Publication Reception of Research}

Altmetrics can help researchers demonstrate the impact of their research, in particular if the research outputs are not journal articles, but datasets, software, etc., and if the impact is best demonstrated in metrics other than citations. ImpactStory ${ }^{6}$ focuses on this use case. Often creators of web-native scholarly products like datasets, software, and blog posts are hard pressed to demonstrate the impact of their work, given a reward system built for a paper-based scholarly publishing world. In these cases, ImpactStory helps to provide data to establish the impacts of these products and allow forward-thinking researcher. ImpactStory also gathers altmetrics to demonstrate wider impacts of traditional products, tracking their impact through both traditional citations and novel altmetrics.

\footnotetext{
5 Altmetric: http://www.altmetric.com/demos/plos.html

6 ImpactStory: http://impactstory.org
} 


\section{Business Intelligence for a Journal, University or Funder}

The focus is not on the individual article, but rather on overall trends over time and/or across funding programs, disciplines, etc. This is an area that the typical researchers is usually less interested in, but is important for strategic decisions by departments, universities, funding organizations, publishers, and others. This area has been dominated by large commercial bibliographic databases such as Web of Science or Scopus, using citation data. Plum Analytics ${ }^{7}$ is a new service that also provide altmetrics and is focusing on universities. The publisher $\operatorname{PLOS}^{8}$ makes a comprehensive set of citations, usage data and altmetrics available for all articles they published.

\section{Altmetrics as an Evaluation Tool}

Traditional scholarly metrics are often used as an evaluation tool, including inappropriate uses such as using the Journal Impact Factor to evaluate publications of individual researchers. Before altmetrics can be used for evaluation, the following questions need to be addressed:

- Can numbers reflect the impact of research, across disciplines and over time?

- Does the use of metrics for evaluation create undesired incentives?

- Do the currently available altmetrics really measure impact or something else?

- How can we standardize altmetrics?

- How easily can altmetrics be changed by self-promotion and gaming?

The first two questions relate to more general aspects of using scientometrics for evaluation, whereas the last three questions are more specific for altmetrics. All these issues can be solved, but it will probably take some time before altmetrics can be reasonably used for evaluation.

Author-level metrics can also include citations and usage stats. Citations are a more established metric for impact evaluation, and citations based on individual articles are much more meaningful than the metrics for the journal that a researcher has published in. The Hirsch-Index (or h index, Hirsch 2005) is a popular metric to quantify an individual's scientific research output. The $h$ index is defined as the number of papers with citation number $\geq h$, e.g. an $\mathrm{h}$ index of 15 means a researcher has published at least 15 papers that have been cited at least 15 times.

\footnotetext{
7 Plum Analytics: http://www.plumanalytics.com

8 PLOS Article-Level Metrics: http://article-level-metrics.plos.org
} 


\section{Example Metrics and Providers}

A growing number of metrics are used by the altmetrics community, and the most important metrics and providers are listed below. Not all metrics measure scholarly impact, some of them are indicators of attention, and in rare cases selfpromotion. Some metrics are good indicators of activity by scholars (e.g. citations or Mendeley bookmarks), whereas other metrics reflect the attention by the general public (e.g. Facebook or HTML views) (Table 1).

Metrics describe different activities: usage stats look at the initial activity of reading the abstract and downloading the paper, whereas citations are the result of much more work, they therefore account for less than $0.5 \%$ of all HTML views. Altmetrics tries to capture the activities that happen between viewing a paper and citing it, from saving an article to informal online discussions.

\section{Mendeley}

Mendeley is one of the most widely used altmetrics services-the number of articles with Mendeley bookmarks is similar to the number of articles that have ciations. Mendeley provides information about the number of readers and groups. In contrast to CiteULike no usernames for readers are provided, but Mendeley provides basic information regarding demographics such as country and academic position. Mendeley is a social bookmarking tool used by scholars and the metrics probably reflect an important scholarly activity_adding a downloaded article to a reference manager.

\section{CiteULike}

CiteULike is another social bookmarking tool, not as widely used as Mendeley and without reference manager functionality. One advantage over Mendeley is that usernames and dates for all sharing events are publicly available, making it easier to explore the bookmarking activity over time.

Table 1 Categorizing metrics into target audiences and depth of interaction (cf. ImpactStory 2012)

\begin{tabular}{lll}
\hline & Scholars & Public \\
\hline Discussed & Science blogs, journal comments & Blogs, Twitter, Facebook, etc. \\
Recommended & Citations by editorials, Faculty of 1,000 & Press release \\
Cited & Citations, full-text mentions & Wikipedia mentiones \\
Saved & CiteULike, Mendeley & Delicious, Facebook \\
Viewed & PDF downloads & HTML views \\
\hline
\end{tabular}




\section{Twitter}

Collecting tweets linking to scholarly papers is challenging, because they are only stored for short periods of time (typically around 7 days). There is a lot of Twitter activity around papers, and only a small fraction is from the authors and/or journal. With some journals up to $90 \%$ of articles are tweeted, the number for new PLOS journal articles is currently at about $50 \%$. The Twitter activity typically peeks a few days after publication, and probably reflects attention rather than impact.

\section{Facebook}

Facebook is almost as popular as Twitter with regards to scholarly content, and provides a wider variety of interactions (likes, shares and comments). Facebook activity is a good indicator for public interest in a scholarly article and correlates more with HTML views than PDF downloads.

\section{Wikipedia}

Scholarly content is frequently linked from Wikipedia, covering about $6 \%$ of all journal articles in the case of PLOS. The Wikipedia Cite-o-Meter ${ }^{9}$ by Dario Taraborelli and Daniel Mietchen calculates the number of Wikipedia links per publisher. In the English Wikipedia the most frequently cited publisher is Elsevier with close to 35,000 links. In addition to Wikipedia pages, links to scholarly articles are also found on user and file pages.

\section{Science Blogs}

Blog posts talking about papers and other scholarly content are difficult to track. Many science bloggers use a blog aggregator, Research Blogging, Nature Blogs and ScienceSeeker being the most popular ones. The number of scholarly articles discussed in blog posts is small (e.g. less than $5 \%$ of all PLOS articles), but they provide great background information and can sometimes generate a lot of secondary activity around the original paper (both social media activity and downloads).

\footnotetext{
9 Wikipedia Cite-o-Meter: http://toolserver.org/ dartar/cite-o-meter/
} 


\section{Altmetrics Service Providers}

Comprehensive altmetrics are currently only available from a small number of service providers. This will most likely change in the near future, as more organizations become interested both in analyzing altmetrics for their content (publishers, universities, funders) or for providing altmetrics as a service.

The Open Access publisher Public Library of Science (PLOS) was the first organization to routinely provide altmetrics on a large number of scholarly articles. The first version of their article-level metrics service was started in March 2009, and PLOS currently provides usage data, citations and social web activity from 13 different data sources. The article-level metrics data are provided via an open $\mathrm{API}^{10}$ and as monthly public data dump.

Altmetric.com is a commercial start-up that started in July 2011. They maintain a cluster of servers that watch social media sites, newspapers and magazines for any mentions of scholarly articles. The data are available to individual users and as service for publishers.

ImpactStory is a non-profit service providing altmetrics since late 2011. They provide both altmetrics and traditional (citation) impact metrics for both traditional and web-native scholarly products, and are designed to help researchers better share and be rewarded for their complete impacts.

Plum Analytics is a start-up providing altmetrics data to universities and libraries. They also provide usage stats and citation data, and track research outputs beyond journal articles, e.g. presentations, source code and datasets.

At this time it is unclear how the altmetrics community will develop over the next few years. It is possible that one or a few dominant commercial players emerge similar to the market for citations, that a non-profit organization is collected these numbers for all stakeholders, or that we see the development of a more distributed system with data and service providers, similar to how usage data for articles are distributed.

\section{Challenges and Criticism}

Many challenges remain before we can expect altmetrics to be more widely adopted. A big part of the challenge is the very nature of the Social Web, which is much more difficult to analyze than traditional scholarly citations.

1. the constantly changing nature of the Social Web, including the lack of commonly used persistent identifiers

2. self-promotion and gaming, inherit to all Social Web activities, and aggravated by the difficulty of understanding who is talking

${ }^{10}$ GitHub: https://github.com/articlemetrics/alm/wiki/API 
3. Altmetrics is more interested in things that can be measured, rather than things that are meaningful for scholarly impact. We therefore measure attention or self-promotion instead of scholarly impact.

These challenges are less of a problem for discovery tools based on altmetrics, but are hard to solve for evaluation tools. Altmetrics is still a young discipline and the community is working hard on these and other questions, including standards, anti-gaming mechanisms, and ways to put metrics into context.

Open Access This chapter is distributed under the terms of the Creative Commons Attribution Noncommercial License, which permits any noncommercial use, distribution, and reproduction in any medium, provided the original author(s) and source are credited.

\section{References}

Bar-Ilan, J. (2012). JASIST@mendeley, Available at: http://altmetrics.org/altmetrics12/bar-ilan/. Eysenbach, G. (2011). Can Tweets predict citations? metrics of social impact based on Twitter and correlation with traditional metrics of scientific impact. Journal of Medical Internet Research, 13(4), p.e123. doi:10.2196/jmir.2012.

Groth, P., \& Gurney, T. (2010). Studying scientific discourse on the web using bibliometrics: A chemistry blogging case study. In Proceedings of the WebSci10: Extending the Frontiers of Society On-Line, April 26-27th 2010. Raleigh, NC, USA. Available at: http:// journal.webscience.org/308/.

Smith, R. (2011). What is post publication peer review? BMJ Group Blogs. Available at: http:// blogs.bmj.com/bmj/2011/04/06/richard-smith-what-is-post-publication-peer-review/.

Hirsch, J.E. (2005). An index to quantify an individual's scientific research output. Proceedings of the National Academy of Sciences, 102(46), pp.16569-16572. doi:10.1073/pnas. 0507655102 .

ImpactStory, (2012). A new framework for altmetrics. ImpactStory blog. Available at: http:// blog.impactstory.org/2012/09/14/31524247207/.

Letierce, J. et al. (2010). Using Twitter during an academic conference: The \#iswc2009 UseCase. In Proceedings of the Fourth International AAAI Conference on Weblogs and Social Media. Available at: http://www.aaai.org/ocs/index.php/ICWSM/ICWSM10/paper/view/1523.

Li, X., \& Thelwall, M. (2012). F1000, Mendeley and traditional bibliometric indicators. Available at: http://sticonference.org/Proceedings/vol2/Li_F1000_541.pdf.

Li, X., Thelwall, M., \& Giustini, D. (2011). Validating online reference managers for scholarly impact measurement. Scientometrics, 91(2), 461-471. doi:10.1007/s11192-011-0580-x.

Neylon, C., \& Wu, S. (2009). Article-Level metrics and the evolution of scientific impact. PLoS Biology, 7(11), p.e1000242. doi:10.1371/journal.pbio.1000242.

Nielsen, F.A. (2007). Scientific citations in Wikipedia. First Monday, 12(8). Available at: http:// firstmonday.org/article/view/1997/1872.

Priem, J. et al. (2010). altmetrics: a manifesto. altmetrics. Available at: http://altmetrics.org/ manifesto/.

Priem, J., \& Costello, K.L. (2010b). How and why scholars cite on Twitter. Proceedings of the American Society for Information Science and Technology, 47(1), pp.1-4. doi:10.1002/ meet.14504701201.

Priem, J., Costello, K.L., \& Dzuba, (2011). Poster at Metrics 2011: Symposium on Informetric and Scientometric Research. 
Priem, J., \& Hemminger, B.M. (2010). Scientometrics 2.0: Toward new metrics of scholarly impact on the social web. First Monday, 15(7). Available at: http://firstmonday.org/htbin/ cgiwrap/bin/ojs/index.php/fm/article/view/2874/2570.

Priem, J., Groth, P., \& Taraborelli, D. (2012a). The Altmetrics Collection Ouzounis C. A. (Ed.), PLoS ONE, 7(11), p.e48753. doi:10.1371/journal.pone.0048753.

Priem, J., Piwowar, H.A., \& Hemminger, B.M. (2012b). Altmetrics in the wild: Using social media to explore scholarly impact. Available at: arXiv:1203.4745v1.

Shema, H., Bar-Ilan, J., \& Thelwall, M. (2012). Research Blogs and the discussion of scholarly information Ouzounis C. A. (Ed.), PLoS ONE, 7(5), p.e35869. doi:10.1371/journal. pone.0035869.

Taraborelli, D. (2008). Soft peer review: social software and distributed scientific evaluation. In P. Hassanaly et al. (Eds.), Proceedings of the 8th International Conference on the Design of Cooperative Systems, Carry-le-Rouet. Aix-en-Provence, France: Institut d'Etudes Politiques d'Aix-en-Provence, pp.99-110.

Weller, K., \& Peters, I. (2012). Citations in Web 2.0. Available at: http://nfgwin.uniduesseldorf.de/sites/default/files/Weller.pdf.

Weller, K., \& Puschmann, C. (2011). Poster presented at the 3rd International Conference on Web Science (ACM WebSci'11), Available at: http://files.ynada.com/posters/websci11.pdf. 